predictive of recovery in function following acute coronary syndromes. Therefore, coronary wave intensity analysis may be a useful adjunctive tool during cardiac catheterisation in the assessment of viability following ACS.

\section{THE ROLE OF CARDIOVASCULAR MRI IN TROPONIN POSITIVE ACUTE CORONARY SYNDROMES WITH UNOBSTRUCTED CORONARY ARTERIES}

doi:10.1136/heartjnl-2012-301877b.134

E McAlindon, * A G Porto, A Chiarellli, G Cincotta, C Lawton, C Bucciarelli-Ducci. Bristol Heart Institute, Bristol, UK

Background Patients with acute coronary syndrome and unobstructed coronary arteries represent a clinical dilemma in whom clinical management is uncertain. Cardiovascular magnetic resonance (CMR) has the potential to non-invasively identify the presence of myocardial infarction or acute myocarditis, thus establishing a final diagnosis with management implications.

Aim To assess the diagnostic value of CMR in patients presenting with ACS and unobstructed coronary arteries.

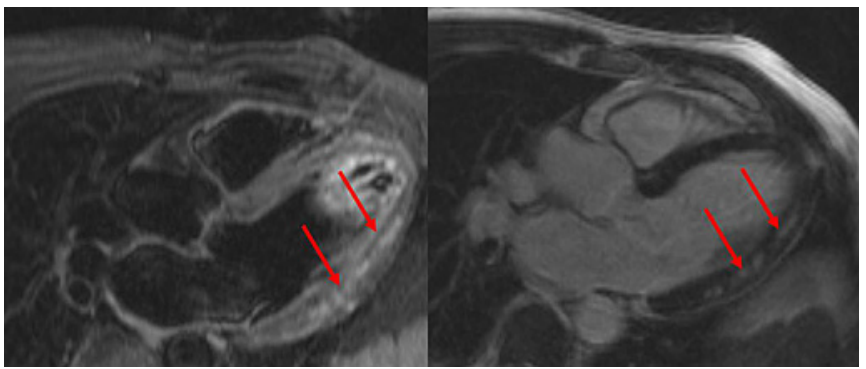

Abstract 134 Figure 1 Acute myocarditis with mid myocardial and epicardial oedema and fibrosis.

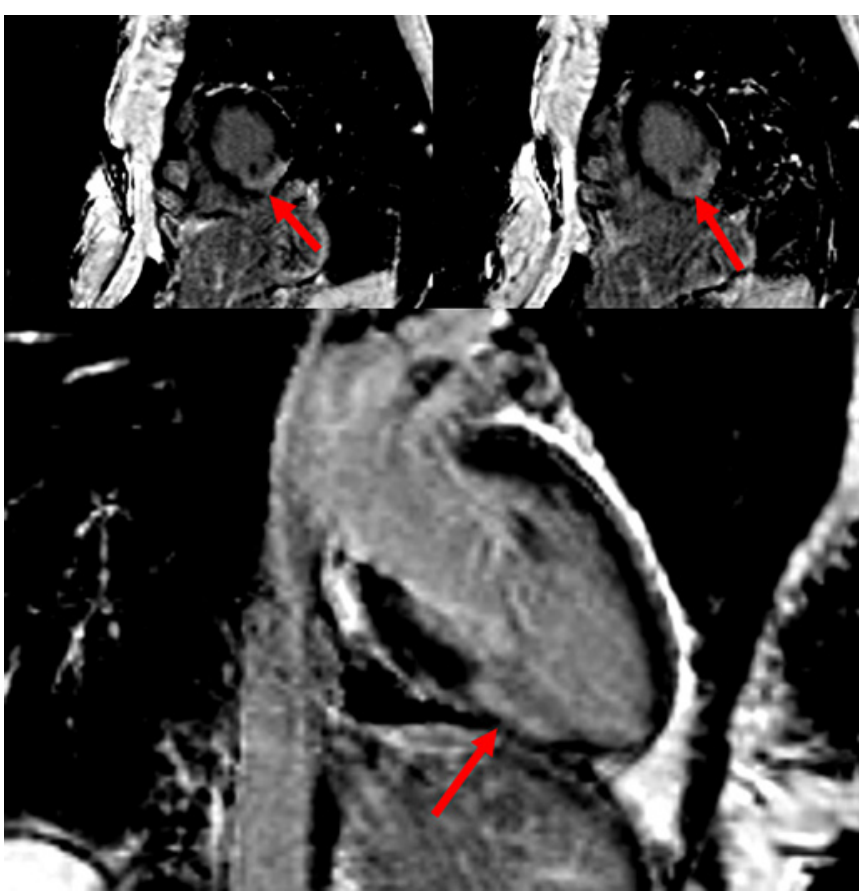

Abstract 134 Figure 2 Acute myocardial infarction with late gadolinium enhancement in the mid-cavity and apical inferior walls.
Methods From October 2010 to November 2011, 48 patients who presented with troponin positive ACS and unobstructed coronary arteries were consecutively recruited. A comprehensive CMR protocol, including T2 weighted STIR imaging for oedema and late gadolinium enhancement imaging for myocardial scarring, was performed within 4 weeks of the index event.

Results In $75 \%$ of cases, a cause for the troponin rise was found. Based on the oedema and scarring patterns observed, the most common diagnoses were acute myocarditis (Abstract 134 figure 1) in $40 \%$ of cases and acute myocardial infarction with spontaneous coronary recanalisation/embolus (Abstract 134 figure 2) in 19\% of the cases. In six patients (12\%) a diagnosis of cardiomyopathy was established: dilated cardiomyopathy $(n=3)$, hypertrophic cardiomyopathy $(n=1)$ and Tako-Tsubo cardiomyopathy $(n=2)$. Acute pericarditis was present in two patients (4\%). The remaining $25 \%$ of patients had a normal CMR scan.

Conclusion In the setting of acute coronary syndromes with unobstructed coronary arteries CMR was able to establish a final diagnosis in $75 \%$ of patients, identifying acute myocarditis, myocardial infarction with spontaneous recanalisation/embolus, and cardiomyopathies. Establishing a final diagnosis has an important impact in patient management and secondary prevention.

\section{CHRONIC DPP-4 INHIBITION BY SITAGLIPTIN ENHANCES BOTH GLOBAL AND REGIONAL MYOCARDIAL FUNCTION DURING DOBUTAMINE STRESS IN PATIENTS WITH TYPE 2 DIABETES MELLITUS AND CORONARY ARTERY DISEASE}

doi:10.1136/heartjnl-2012-301877b.135

L M McCormick,* A C Kydd, P A Read, S P Hoole, D P Dutka. Department of Cardiovascular Medicine, University of Cambridge, Cambridge, UK

Background Glucagon-like peptide-1 (GLP-1) is an incretin hormone secreted by the gut in response to the oral ingestion of nutrients. Through augmentation of myocardial glucose uptake and subsequent reduction in fatty acid oxidation, GLP-1 modulation therapy has emerged as a potential target for improving myocardial oxygen efficiency at times of ischaemic stress, such as occurs in obstructive coronary artery disease (CAD). Sitagliptin is a DDP-4 inhibitor licensed for the treatment of Type 2 Diabetes Mellitus (T2DM) that reduces degradation of plasma GLP-1 (7-36). We investigated whether sitagliptin improved myocardial performance during dobutamine stress echocardiography (DSE) in patients with T2DM and $\mathrm{CAD}$

Methods 12 patients (aged 69 \pm 9 years, 9 men) with T2DM on oral hypoglycaemic therapy (OHT), obstructive $\mathrm{CAD}$ and preserved left ventricular (LV) systolic function were studied. Each subject underwent DSE on two separate occasions after an overnight fast; the first (control) while receiving standard OHT and the second after the addition of sitagliptin (100 mg od) for 4 weeks. Tissue Doppler imaging was acquired in three apical views at rest, peak stress and 30 min recovery. Global function was assessed by ejection fraction (EF) using the Simpson's biplane method and mitral annular peak systolic velocity (MASV) averaged over six sites. Regional LV wall motion was assessed using a 12-segment model comprising the base and mid-level of six regional walls. Peak systolic tissue velocity (Vs), strain (S) and strain rate (SR) were calculated for each region from tissue Doppler velocity data averaged over three consecutive beats using an off-line workstation (EchoPac, GE Medical Systems).

Results At rest, all parameters of both global and regional LV performance were unchanged after sitagliptin. At peak stress, the rate-pressure product attained was the same for both DSE studies but those after sitagliptin demonstrated significantly enhanced myocardial performance in both global (LVEF $69.9 \pm 6.5$ vs $63.9 \pm 6.2 \%, \quad p=0.001 ;$ MASV $12.69 \pm 3.0$ vs $11.65 \pm 3.5 \mathrm{~cm} / \mathrm{s}$, 
$\mathrm{p}=0.003$ ) and regional parameters (data for 12 segments; vs $10.8 \pm 4.4$ vs $9.9 \pm 4.4 \mathrm{~cm} / \mathrm{s}, \mathrm{p}<0.0001 ; \mathrm{S}-15.7 \pm 4.7$ vs $-14.8 \pm 4.4 \%$, $\mathrm{p}=0.03$; SR $-2.2 \pm 0.5 \mathrm{vs}-2.0 \pm 0.5 / \mathrm{s}, \mathrm{p}<0.0001)$. The improvement was greater in those regions subtended by a coronary artery with $>50 \%$ stenosis than in those supplied by a non-obstructed artery. At 30 min recovery, ischaemic LV dysfunction did not occur after sitagliptin (LVEF $55.6 \pm 3.5$ vs $49.7 \pm 2.6 \%, \quad \mathrm{p}=0.0001 ; \mathrm{MASV}$ $5.55 \pm 1.2$ vs $5.20 \pm 1.0 \mathrm{~cm} / \mathrm{s}, \mathrm{p}=0.003)$.

Conclusion In patients with CAD and T2DM receiving OHT, DPP-4 inhibition by sitagliptin improves both global and regional myocardial performance during demand ischaemia (with greater benefit seen in ischaemic vs non-ischaemic segments), and mitigates post-ischaemic stunning.

\section{INCREASE IN LEFT VENTRICULAR MASS IN TYPE 2 DIABETES IS DEPENDENT ON DURATION OF DIABETES}

doi:10.1136/heartjnl-2012-301877b.136

${ }^{1}$ A K Ghosh, ${ }^{* 1}$ A D Hughes, ${ }^{1} \mathrm{~N}$ Chaturvedi, ${ }^{1} \mathrm{D}$ P Francis, ${ }^{2} \mathrm{D}$ Pellerin, ${ }^{3} \mathrm{~J}$ Deanfield, ${ }^{4} \mathrm{M}$ Pierce, ${ }^{4} \mathrm{D}$ Kuh, ${ }^{1} \mathrm{~J}$ Mayet, ${ }^{4} \mathrm{R}$ J Hardy. ${ }^{1}$ International Centre for Circulatory Health, Imperial College, London, UK; ${ }^{2}$ The Heart Hospital, London, UK; ${ }^{3}$ Institute of Child Health, University College London, London, UK; ${ }^{4}$ Medical Research Council Unit for Lifelong Health and Ageing, London, UK

Background Adverse consequences of type 2 diabetes mellitus (T2DM) include an increase in left ventricular mass (LVM). Whether the underlying mechanisms of this association are due to the hyperglycaemic state per se, or to other risk factors is unclear. We explored the association between diabetes duration, an index of the chronicity of the hyperglycaemic state, with LVM.

Methods The Medical Research Council National Survey of Health and Development is a birth cohort of men and women born in Britain in 1 week in March 1946. When study members were 60-64 years of age, 1700 underwent echocardiography according to ASE guidelines and LVM indexed to height 2.7 (LVMI) was calculated. Date of diagnosis of T2DM by a doctor was obtained in the 175 cohort members reporting T2DM through information supplied on postal questionnaires and at interview. Linear regression models were fitted to estimate the effects of presence and of duration of diabetes on LVMI at age 60-64 years.

Results In a linear regression model adjusted for sex, current body mass index (BMI), and current systolic blood pressure (SBP), LVMI increased by $0.78 \mathrm{~g} / \mathrm{m}^{2}$ for every year increase in diabetes duration. When diabetes duration was divided into categories, this same trend was observed. Compared with those with no diagnosis of diabetes, LVMI was $3.82 \mathrm{~g} / \mathrm{m}^{2}$ (95\% CI 0.73 to $\left.6.93, \mathrm{p}=0.02\right)$ higher for $0-10$ years duration of diabetes, $7.46 \mathrm{~g} / \mathrm{m}^{2}(95 \% \mathrm{CI}-1.94$ to 16.87 , $\mathrm{p}=0.12)$ higher for $10-20$ years duration, and $17.28 \mathrm{~g} / \mathrm{m}^{2}(95 \%$ CI 1.01 to 33.56, $\mathrm{p}=0.04$ ) higher for $20-30 \mathrm{y}$ duration of diabetes.

Conclusions LVMI increases significantly with diabetes duration, even when other major risk factors are accounted for. As T2DM is increasingly being diagnosed at younger ages, this duration effect has important implications for the future burden of associated cardiac disease, and indicates that hyperglycaemia per se plays a key role in the adverse consequences of T2DM on the left ventricle. This also has implications for current treatment and prevention strategies.

\section{7 \\ TIME FOR A REVIEW OF THE "WATCH AND WAIT" STRATEGY FOR YOUNG BORDERLINE-HYPERTENSIVES?}

doi:10.1136/heartjnl-2012-301877b.137

${ }^{1} \mathrm{~A}$ K Ghosh, ${ }^{*} \mathrm{R}$ J Hardy, ${ }^{1} \mathrm{D}$ P Francis, ${ }^{1} \mathrm{~N}$ Chaturvedi, ${ }^{3} \mathrm{D}$ Pellerin, ${ }^{4} \mathrm{~J}$ Deanfield, ${ }^{2} \mathrm{D}$ Kuh, ${ }^{1} \mathrm{~J}$ Mayet, ${ }^{1} \mathrm{~A} D$ Hughes. ${ }^{1}$ International Centre for Circulatory Health, Imperial
College, London, UK; ${ }^{2}$ Medical Research Council Unit for Lifelong Health and Ageing, London, UK; ${ }^{3}$ The Heart Hospital, London, UK; ${ }^{4}$ Institute of Child Health, University College London, London, UK

Background In cross-sectional studies, elevated systolic blood pressure (SBP) is associated with increased left ventricular mass (LVM), which leads to increased cardiovascular morbidity and mortality. Current guidelines recommend a "watch and wait" approach in younger individuals with a borderline-high SBP. We investigated if this was a safe assumption.

Methods The Medical Research Council National Survey of Health and Development is a birth cohort study following men and women born in Britain in 1 week in March 1946. When study members were 60-64 years of age, 1700 underwent echocardiography and LVM indexed to body-surface-area (LVMI) was measured. The relationship between repeated measures of SBP and antihypertensive treatment (measured at 4 time points: ages 60-64 (current), 53, 43 and 36) and LVMI at 60-64 years was examined using sex-adjusted multiple regression models. Then, multilevel models of SBP were used to estimate person-specific intercepts (SBP at age 36) and slopes (rate of change in SBP between 36 and 53 years). The intercepts and slopes were then included in sex-adjusted linear regression models with LVMI as the outcome.

Results Individuals on treatment for hypertension, from age 43 years onwards, had higher mean LVMI than those who were not on treatment, irrespective of level of SBP at the same age. LVMI was $12.3 \mathrm{~g} / \mathrm{cm}^{2}$ (95\% CI 9.2 to $15.4 ; \mathrm{p}<0.001$ ) higher for those currently on treatment, $10.0 \mathrm{~g} / \mathrm{cm}^{2}$ higher (95\% CI 5.9 to $14.1 ; \mathrm{p}<0.001$ ) for those on treatment at age 53 , and $15.1 \mathrm{~g} / \mathrm{cm}^{2}$ higher $(95 \%$ CI 5.8 to 24.3; $\mathrm{p}=0.001$ ) for treatment at age 43 . In associated analyses, the effect of mid-life rate of change in SBP (from 36 to 53 years) on LVMI at age 60-64 years was 10 times greater than the effect of more recent rate of change in SBP (from 53 years to current).

Conclusions Our research suggests being on antihypertensive treatment may not normalise LVMI due to irreversible cardiac damage occurring in mid-life in poorly controlled hypertensives. Early identification and effective treatment of individuals with rapidly increasing SBP in mid-life may be key to preventing such damage. A review of current guidelines on monitoring and screening of blood pressure may thus be required.

\section{NON-ANGINAL CHEST PAIN: NOT AS BENIGN AS WE WOULD LIKE TO BELIEVE!}

doi:10.1136/heartjnl-2012-301877b.138

A George, ${ }^{*}$ T Anwar, K Advani, D Cullington, J John, S Chattopadhyay. Scunthorpe General Hospital, Scunthorpe, UK

Background In the Quick Reference Guide of the clinical guidance 95 (CG95) NICE recommends that chest pain (CP) diagnosed as nonanginal (NA) should not be investigated for stable angina routinely. In the Full Guidance, it qualifies this statement suggesting that stable angina should be excluded in patients with NACP unless clinical suspicion is raised based on other aspects of the history and risk factors. In the chest pain management algorithm, however, it excludes patients with NACP in whom stable angina is suspected based on history and risk factors. This study was undertaken to assess the outcome of patients attending rapid access chest pain clinic (RACPC) and diagnosed with NACP who are likely to be discharged without further investigation as suggested by CG95.

Method and Results 1042 consecutive RACPC referrals between November 2009 and April 2011 were reviewed. Demographics, CG95 defined risk factors, CP characteristics, history of confirmed coronary artery disease (CAD), results of the exercise ECG test, management plan and outcomes (composite end point of all cause 